\title{
Avaliação do WRF-4DVAR em um caso de evento severo no Rio Grande do Sul
}

\author{
WRF-4DVAR Evaluation of an extreme event over Rio Grande do Sul
}

\author{
Luana Ribeiro Macedo ${ }^{1}$, João Luiz Martins Basso² e Yoshihiro Yamasaki² \\ ${ }^{1}$ Universidade de São Paulo, SP, Brasil \\ lumeteorologia@gmail.com \\ ${ }^{2}$ Universidade Federal de Pelotas, RS, Brasil \\ joobasso@gmail.com; yamasaki07722@gmail.com
}

\begin{abstract}
Resumo
A técnica de assimilação de dados 4DVAR do sistema de mesoescala WRF foi utilizada para avaliar o impacto da assimilação de dados nos prognósticos numéricos do tempo sobre o Estado do Rio Grande do Sul. A assimilação é feita utilizando dados de superfície e altitude e a consistência do processo feita sobre os prognósticos numéricos, explorando as diferenças entre análises com e sem assimilação de dados. Os resultados dos prognósticos, para cada caso avaliado, foram comparados utilizando dados do TRMM e de refletividade do radar meteorológico, instalado em Canguçu. A variável precipitação acumulada foi espacialmente validada com os dados do TRMM, para período de 12 horas, compreendido entre 29 e 30 de outubro de 2014. Foi possível observar que, tanto a simulação com e sem assimilação superestimaram os valores da precipitação. O campo de refletividade do radar, sem assimilação de dados - para as 16:00 UTC de 30 de outubro - detectou com melhor precisão os núcleos de refletividade, sobre o Estado do Rio Grande do Sul. A análise dos campos da temperatura revelaram que o sistema de assimilação 4DVAR contribui, de uma maneira ou outra, apresentando prognósticos com uma pequena melhora comparados com os dados observados em alguns pontos.
\end{abstract}

Palavras-chave: Assimilação de Dados, WRF, TRMM.

\begin{abstract}
The WRF mesoscale system 4DVAR data assimilation technique have been used with the purpose of evaluating the impact of the meteorological data assimilation on the numeric time prognosis over the Rio Grande do Sul state. It has been done utilizing the surface and altitude data. The consistency analysis has been done evaluating the numerical prognosis exploring the differences between the analysis with and without data assimilation. The produced prognosis results have been compared spatially using the TRMM satellite data as well as the Canguçu radar reflectivity data. The accumulated rainfall has been validated and compared spatially with the TRMM data for the time period of 12 hours comprehended between October $29^{\text {th }}$ and $30^{\text {th }}$ of 2014. It was possible to realize that as well as the WRF, the WRFVAR overestimated the rainfall values. The radar reflectivity field without data assimilation for October $30^{\text {th }}$ at 06:00UTC detected most accurately the reflectivity centers over the state. On the other hand this field with data assimilation did not present good skill. The temperature field analyses reveal that the 4DVAR assimilation system contributes, one way or another, presenting a little improvement for some points compared to the real data.
\end{abstract}

Keywords: Data Assimilation, WRF, TRMM. 


\section{Introdução}

A habilidade de observar e modelar a atmosfera melhorou significativamente nos últimos anos. Os melhoramentos ocorridos estão diretamente ligados ao aumento da potência dos supercomputadores que vem permitindo o uso de melhores resoluções espacial e temporal, bem como aproximações numéricas menores nos modelos operacionais atmosféricos. Como resultado desses avanços tem-se alcançado um melhor entendimento de diversos processos atmosféricos e do desenvolvimento dos modelos de Previsão Numérica do Tempo (PNT).

Os modelos de PNT contribuem principalmente para a avaliação da intensidade dos sistemas termo hidrodinâmicos atuantes na atmosfera. Entretanto, mesmo com o avanço do sistema computacional, bem como das novas metodologias e incorporações de técnicas avançadas de tratamento físico dos processos atmosféricos, a PNT ainda apresenta algumas falhas. Grande parte dessas falhas se deve às condições iniciais e de fronteira. Assim sendo, ressalta-se que a representação imprecisa do estado inicial da atmosfera resulta num erro na análise inicial que, consequentemente, causam drásticos erros nos resultados - porque as imprecisões introduzidas no sistema podem crescer ao longo do tempo e apresentar previsões, com uma margem de erro maior que o esperado (Kalnay, 2003).

As observações atmosféricas incluem as medidas diretas (in situ) e estimadas por satélite, de variáveis atmosféricas tais como, velocidade do vento, temperatura, umidade e pressão. A combinação dessas variáveis proporciona uma extensiva base de dados para inicialização e verificação dos modelos de PNT, porém não há nenhum sistema capaz de fornecer todas essas variáveis atmosféricas com as mesmas coberturas temporal e espacial e com a precisão necessária. Tampouco existe um sistema de observação que forneça uma distribuição uniforme destas medidas.

Uma solução para extrair o máximo de informações de cada sistema de observação e minimizar os erros das condições iniciais é a técnica de assimilação de dados. Esta técnica utiliza o máximo de observações disponíveis, considerando seus erros, para gerar a análise. A análise representa o estado da atmosfera coerente, não somente com respeito às observações, como também com a dinâmica da atmosfera - gerada em instantes anteriores fornecidos pelo modelo prognóstico. Esse processo de assimilação de dados é feito com o auxílio de um campo de background (ou de suporte), que é gerado a partir de uma simulação feita anteriormente, utilizando um modelo numérico, para o instante das observações. Minimizando assim a diferença entre o campo background e as observações, é gerada então uma representação ótima do estado da atmosfera, considerando suas respectivas

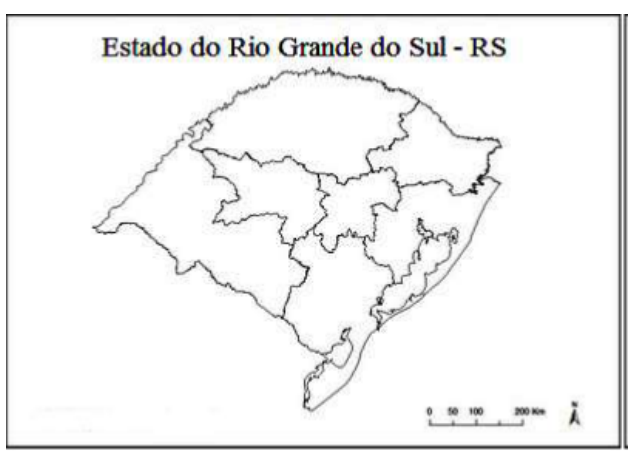

Figura 1: Área de estudo.

matrizes de erros e suas correlações (Kalnay, 2003).

Muitas são as técnicas de assimilação de dados, porém nos últimos anos aquelas baseadas no método variacional vem ganhando destaque nos grandes centros de meteorologia. Neste trabalho, foi avaliado o impacto da assimilação de dados observados com o emprego do sistema Weather Research and Forecasting (WRF) 4DVAR, para um caso de precipitação intensa ocorrida no Estado do Rio Grande do Sul - RS. A peculiaridade do caso explorado está no fato de que, em um período de seis horas, várias cidades a oeste e sul do Estado tiveram precipitações acumuladas bem significativas.

\section{Dados e Metodologia}

A área de estudo envolve todo Estado do Rio Grande do Sul - localizado entre as latitudes $27^{\circ} 03^{\prime} 42^{\prime \prime} \mathrm{S}$ e $33^{\circ}$ $45^{\prime} 09^{\prime \prime} \mathrm{S}$ e as longitudes $57^{\circ} 40^{\prime} 57^{\prime \prime} \mathrm{O}$ e $33^{\circ} 45^{\prime} 41^{\prime \prime} \mathrm{O}$ conforme ilustrado na Figura 1.

A análise do evento foi realizada utilizando imagens do satélite GOES - 13 no canal infravermelho, em alta resolução espacial; cartas de superfície do Centro de Hidrografia da Marinha, campos das análises do modelo global - The Global Forecast System (Sistema de Previsão Global - GFS) do National Centers for Environmental Prediction (NCEP), imagens do satélite Missão de Medida de Chuva Tropical (TRMM), bem como os resultados das simulações do modelo WRF e WRFDA. Para processar o modelo WRF foram utilizados os prognósticos do modelo global GFS. Posteriormente foi feita a inserção dos dados das variáveis meteorológicas das estações de superfície do Insituto Nacional de Meteorologia (INMET) de todo o Estado do RS; radiossondagens do Estado do RS (TEMP), dados do tipo SYNOP, METAR, SATOB, SHIP, BUOY, PILOT e AIREP.

\subsection{Sistema de Modelagem e Assimilação de Dados WRF}

O modelo WRF apresenta um sistema de assimilação de dados variacional denominado WRFDA, compreen- 
dendo os módulos 3DVAR e 4DVAR. Neste trabalho é utilizado o 4DVAR. A técnica de assimilação de dados variacional faz um ajuste para minimizar a diferença entre o campo ajustado e o observado em um processo interativo, considerando as hipóteses físicas como restrições impostas ao escoamento para obter um equilíbrio dinâmico. Essa minimização é efetuada através da função custo J, descrita por Lorenc (1986) com base na teoria de probabilidades bayesiana, como:

$$
\begin{array}{r}
J(x)=\frac{1}{2}\left(x-x_{b}\right)^{T} B^{-1}\left(x-x_{b}\right)+ \\
+\frac{1}{2}\left(y_{0}-H\left(x_{b}\right)\right)^{T} R^{-1}\left(y_{0}-H\left(x_{b}\right)\right)
\end{array}
$$

onde, $x$ é a observação, $x_{b}$ é o campo de background, $y_{0}$ é a estimativa inicial, Hé um operador da observação, $B$ é a matriz de covariância de erros de estimativa e $R$ é a matriz de covariância de erros de observação. A matriz covariância do erro $B$ é a parte mais importante do método da análise variacional para determinar a taxa de impacto, distribuição, e relações dos incrementos das análises.

A assimilação 4DVAR é uma generalização da assimilação 3DVAR para manipular as observações que estão distribuídas no tempo. A função custo é a mesma, entretanto o vetor operador de observação não deve ser generalizado, pois fará a inclusão das observações no horário oportuno para a previsão do modelo. O 4DVAR calcula em um modelo de previsão a melhor trajetória que se adapta as observações disponíveis para dentro do erro de observação ao longo de um período de tempo (Figura 2). O erro de observação inclui um subsídio para a resolução finita do modelo. Sabendo que o modelo não pode representar exatamente a atmosfera, uma trajetória não pode ser realizada para um período superior a 12 horas sem fazer correções a ele. Espera-se, então, que utilizando o método 4DVAR uma melhor previsão seja alcançada através da construção de um ajuste melhor ao longo do intervalo de tempo ao fazer as menores correções possíveis e depois extrapolar para frente no tempo. Este método explora a quantidade máxima de dados observados disponíveis para um período inicial de seis horas.

\subsection{Configurações e Processamento}

O modelo de mesoescala WRF, não hidrostático, foi configurado com um domínio, cuja resolução horizontal é de $18 \mathrm{~km}$, espaçamento de grade de $114 \times 95$ pontos na horizontal, 35 níveis sigma na vertical e topo estabelecido em $50 \mathrm{hPa}$, cobrindo a área de integração apresentada na Figura 3. As parametrizações utilizadas no processamento são apresentadas por Macedo et al. (2014). As integrações numéricas foram realizadas para um período de 48 horas, com armazenamento de prognósticos a cada hora.

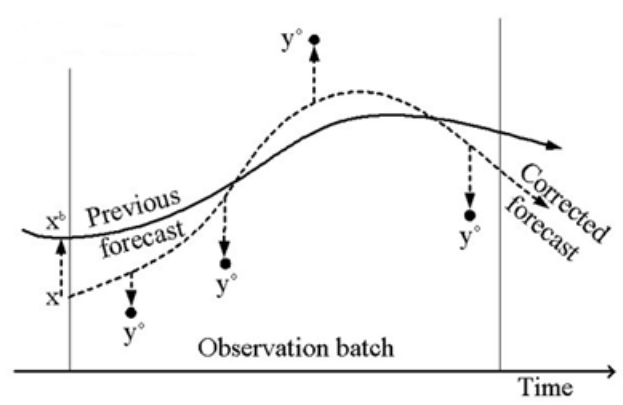

Figura 2: Trajetória de ajuste do sistema 4DVAR. Fonte: (adaptado de Skamarock et al. (2010))

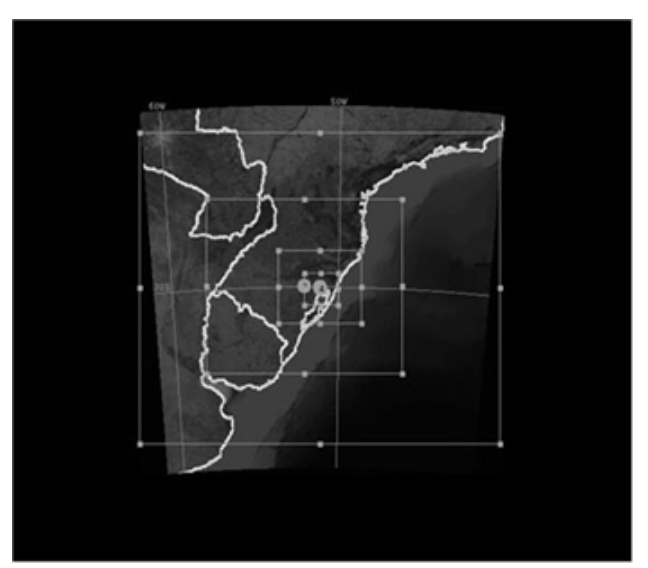

Figura 3: Domínios de Processamento do Modelo WRF.

Os processamentos foram inicialmente realizados a partir do modelo WRF, utilizando como condições iniciais e de contorno, os processamentos do modelo GFS do NCEP. Na sequência, foram realizados processamentos a partir do sistema de assimilação de dados, denominado WRFVAR. A assimilação foi realizada com dados do tipo TEMP, SYNOP, METAR, SATOB, SHIP, BUOY, PILOT, AIREP, e de estações meteorológicas automáticas de superfície do INMET.

\subsection{Radar Meteorológico}

O radar meteorológico utilizado está instalado na cidade de Canguçu- RS. Trata-se de um radar Doppler, localizado à $31^{\circ} 24^{\prime} 14^{\prime \prime} \mathrm{S}$ e $52^{\circ} 42^{\prime} 06^{\prime \prime} \mathrm{W}$, a 466,57 metros de altitude, operando em banda $\mathrm{S}(2,7-3 \mathrm{GHz})$, com comprimento de onda de $10 \mathrm{~cm}$ e abertura do feixe de micro-ondas de aproximadamente $2^{\circ}$. Sua varredura é executada a cada 15 minutos e ele é operado 24 horas por dia. Na Figura 4 é apresentada a área de cobertura do radar de Canguçu, para um raio de $400 \mathrm{Km}$.

Ele é operado pelo Departamento de Controle do Espaço Aéreo (DECEA). No processamento os dados CAPPI ("Constant Altitude Plan Position Indicator") são formatados e armazenados com - 15 níveis verticais - de $2 \mathrm{~km}$ a $16 \mathrm{~km}$. O produto é obtido a partir da refleti- 


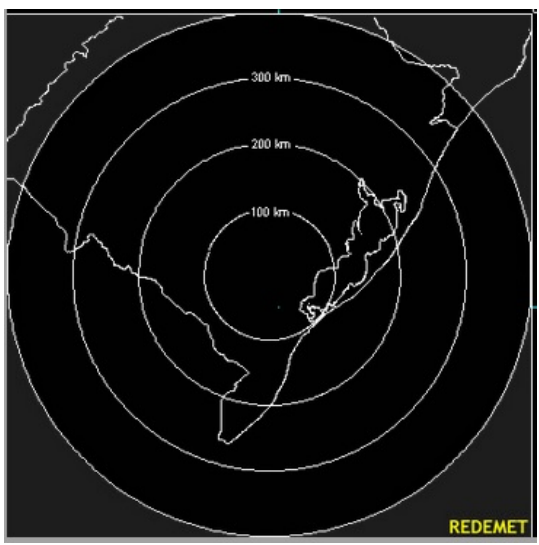

Figura 4: Área de cobertura do radar de Canguçu, para um raio de 400Km. (Fonte: REDEMET (2015))

vidade do radar, coletados de cada azimute e elevação. No final do processo da varredura eletrônica, o volume tridimensional de dados do radar é o que possibilita obter todo o perfil vertical da refletividade do radar em $\mathrm{dBz}$, dentro da área de cobertura. O CAPPI é obtido com os dados da varredura completa do radar, em todas as elevações da antena, na altura desejada. Após fixar a altura, é feita a interpolação das informações, contidas em cada uma das elevações da PPI ("Plan Position Indicator"), formando assim um campo de refletividade em um plano de altitude constante. Como a resolução horizontal dos dados do radar $(1 \mathrm{~km}$ por $1 \mathrm{~km})$ é maior que a do domínio do modelo, eles foram devidamente processados para apresentação compatível com a do modelo.

\subsection{Dados do TRMM}

O uso de satélites na área de previsão do tempo vem crescendo muito nos últimos anos, seja esse para fim de validação da previsão do tempo, ou para a inclusão dos dados na mesma. Nos últimos anos os produtos do TRMM têm sido um dos mais utilizados, tanto para avaliar resultados de modelos numéricos ( Rozante e Cavalcanti (2008); De Gonçalves et al. (2006)), quanto para combinar com dados de estações meteorológicas de superfície ( Rozante et al. (2010); Mitra et al. (2009)). Neste trabalho foi utilizado o produto do TRMM 3B42RT, obtidos com uma resolução temporal e horizontal de 3 horas e $0,25^{\circ}$, respectivamente. O 3B42RT é produzido em tempo quase real, e consiste na combinação dos sensores do TRMM (micro ondas passivo e infravermelho) para estimar a precipitação. Para fins de comparação com as simulações com e sem assimilação de dados, acumulouse os valores do produto 3B42RT para períodos de 12 horas.

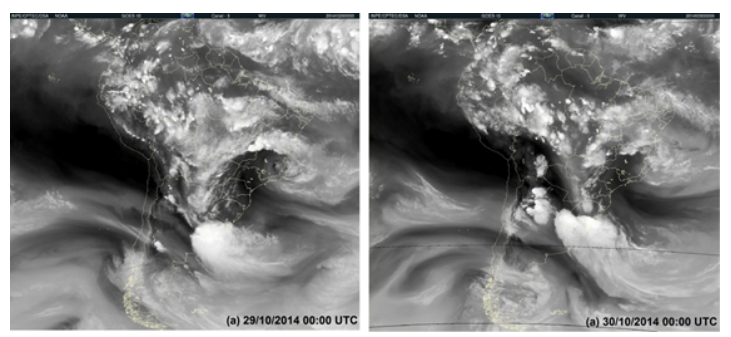

Figura 5: Sequência de imagens do satélite GOES - 13. (FONTE: CPTEC (2015))
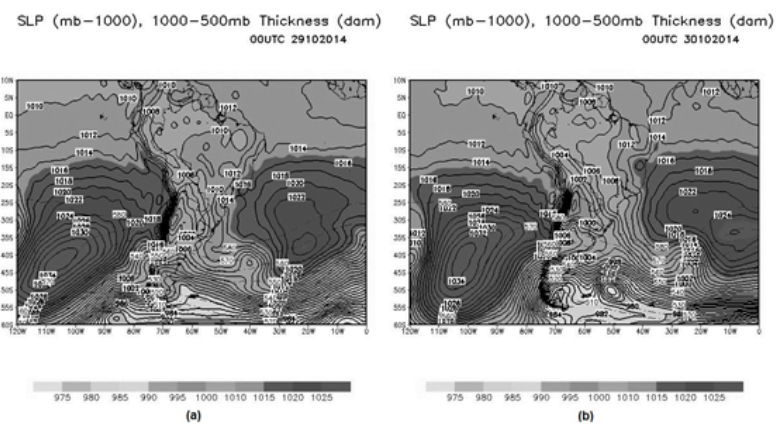

Figura 6: Campo de Pressão ao nível do mar e geopotencial (a) 29/10/2014 00:00 UTC (b) 30/10/2014 00:00 UTC

\section{RESULTADOS E DISCUSSÕES}

Na sequência de imagens do canal infravermelho do satélite GOES - 13, de alta resolução espacial (Figura 5), observa-se o avanço da instabilidade em um período de 24 horas, entre os dias 29 e 30 de outubro de 2015, sobre o Estado do Rio Grande do Sul.

Na Figura 6 são apresentados os campos de pressão em superfície das 00:00 UTC do dia 29/10/2014 (Figura 6-a). A Alta Subtropical do Atlântico Sul (ASAS) atua com um centro de $1024 \mathrm{hPa}$, em torno de $38^{\circ} \mathrm{S} / 25^{\circ} \mathrm{O}$. É possível observar uma frente estacionária que se estende da Argentina (Província San Luís), extremo sul do Uruguai e Atlântico adjacente até se acoplar com uma onda frontal a sudeste $38^{\circ} \mathrm{S} / 48^{\circ} \mathrm{O}$, cujo centro de baixa pressão é de $1004 \mathrm{hPa}$ e localiza-se próximo a $41^{\circ} \mathrm{S} / 40^{\circ} \mathrm{W}$. Ainda é possível observar uma frente fria, entre a Patagônia e o Atlântico, que se acopla a outra nas ilhas Malvinas e a outra a sudeste de $55^{\circ} \mathrm{S} / 50^{\circ} \mathrm{O}$. Outro sistema frontal atua ao sul de $30^{\circ} \mathrm{S}$, com centro de baixa pressão situado em torno de $48^{\circ} / 78^{\circ} \mathrm{O}$. A alta subtropical do Pacífico Sul (ASPS) atua a oeste de $94^{\circ} \mathrm{W}$ com núcleo de $1032 \mathrm{hPa}$. Na análise das 00 UTC do dia 30/10/2014 (Figura 6-b), observa-se um avanço dos sistemas frontais presentes na análise anterior. Sobre o nordeste da Argentina e Uruguai atua uma frente fria, a mesma estende-se pelo Atlântico até um centro de baixa pressão de $976 \mathrm{hPa} e m 50^{\circ} \mathrm{S} / 50^{\circ} \mathrm{O}$. Ao Sul de $40^{\circ} \mathrm{S}$, 

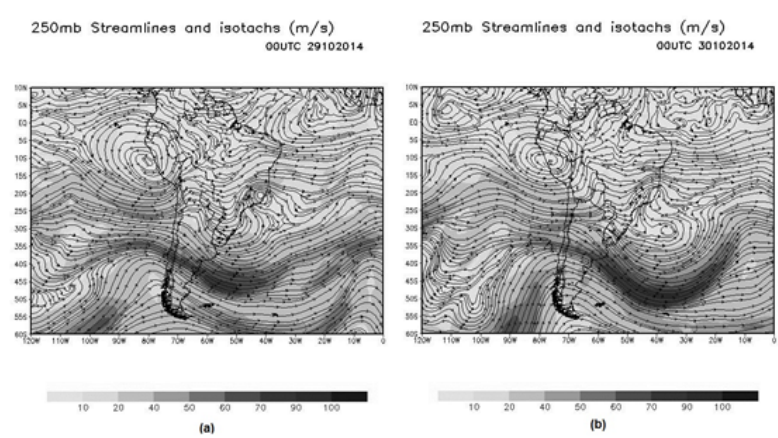

Figura 7: Campo de linhas de corrente e divergência no nível de $250 \mathrm{hPa}$, (a) 29/10/2014 00:00 UTC (b) 30/10/2014 00:00 UTC.

nota-se uma frente fria, que se estende sobre o Chile e o centro da Argentina, com núcleo de pressão de $948 \mathrm{hPa}$ em torno de $50^{\circ} \mathrm{S} / 67^{\circ} \mathrm{O}$. A ASAS atua com centro de $1024 \mathrm{hPa}$ a leste de $20^{\circ} \mathrm{O}$ e a ASPS atua a oeste de $90^{\circ} \mathrm{O}$ com núcleo o valor de $1032 \mathrm{hPa}$.

A carta no nível de $250 \mathrm{hPa}$ da 00:00 UTC do dia 29/10/2015 (Figura 7-a), mostra que o escoamento no continente em latitudes médias se apresenta perturbado com a presença de um Vórtice Ciclônico de Altos Níveis (VCAN), que atua no leste do Sudeste do Brasil em torno de $23^{\circ} \mathrm{S} / 42^{\circ} \mathrm{O}$. Na sua vanguarda o escoamento é difluente e contribui para a nebulosidade convectiva sobre o oceano conforme é possível observar na imagem de satélite (Figura 5-a). Uma crista atua entre o centro de MT e o RS, o que contribui para deixar o tempo sem nuvens nessa grande área. Ao sul deste centro de alta pressão, nota-se a presença dos Jatos Subtropical (JST) e ramos do Jato Polar (JP), entre $32^{\circ} \mathrm{S}$ e $43^{\circ} \mathrm{O}$ no continente. Estes jatos contornam um cavado entre o Pacífico e o continente dando suporte ao sistema frontal em superfície. Um cavado tem seu eixo entre o oeste da Argentina e o leste do Peru e contribui para nebulosidade convectiva isolada. No dia 30/10/2015 às 00:00 UTC (Figura 7-b), observa-se o deslocamento do VCAN apresentado no dia 29/10/2015, o mesmo agora atua no leste do Sudeste do Brasil em torno de $21^{\circ} \mathrm{S} / 41^{\circ} \mathrm{O}$ e continua influenciando o escoamento no continente em latitudes médias. Na sua vanguarda o escoamento é difluente e contribui para a formação da Zona de convergência de Umidade (ZCOU) e nebulosidade convectiva sobre o oceano, conforme se verifica na imagem de satélite da Figura 5-b, também para GO, sul de TO e sul, sudeste e oeste da BA. Uma crista atua entre o centro de MT e SC. Esse sistema contribui para deixar o tempo sem nuvens nesta grande área. A sudoeste/leste desta crista há presença de um cavado, entre RO e o oeste do RS. A sul deste centro anticiclônico, nota-se a presença dos Jatos Subtropical (JST) praticamente inexistente do continente e dos ramos norte e sul do Polar (JPN e JPS),

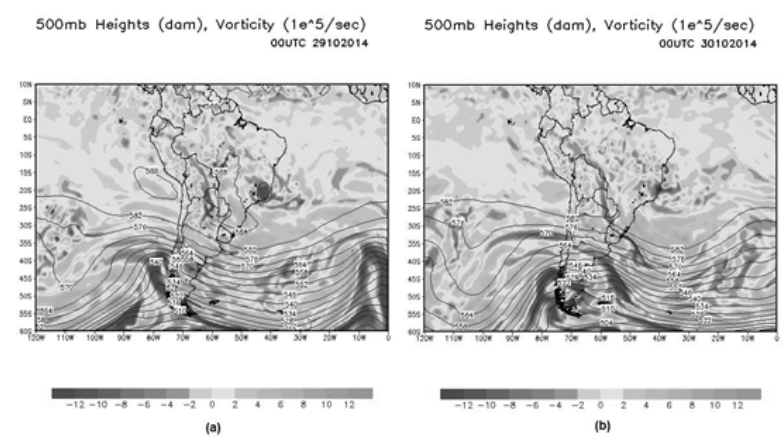

Figura 8: Campo de vorticidade relativa e linha de corrente em $500 \mathrm{hPa}$, (a) 29/10/2014 00:00 UTC (b) 30/10/2014 00:00 UTC.

entre $34^{\circ} \mathrm{S}$ e $44^{\circ} \mathrm{O}$ no continente. Estes jatos contornam um cavado entre o Pacífico e o continente dando suporte ao sistema frontal em superfície, sobre o Uruguai.

No campo de vorticidade e linhas de corrente no nível de $500 \mathrm{hPa}$ das 00UTC do dia 29/10/2015 (Figura 8-a), é possível observar que grande parte do continente é influenciado por uma ampla área anticiclônica, onde o centro atua no leste da Região Sul. Este sistema gera subsidência do ar e dificulta a formação de nebulosidade na Argentina, Uruguai e na Região Sul. Observa-se uma zona de grande baroclinia no Pacífico, na qual atuam ventos fortes e de intenso gradiente de geopotencial entre $30^{\circ} \mathrm{S}$ e $50^{\circ} \mathrm{S}$, evidenciada pela presença de um cavado frontal. Esta zona também atinge áreas da Patagônia e leste da Argentina. Um cavado atua entre o centro da Argentina e o sul da Bolívia, que favorece o levantamento do ar gerando nebulosidade convectiva em algumas áreas entre o centro da Argentina e a Bolívia. Um Vórtice Ciclônico é visto no leste do Sudeste do Brasil em torno de $21^{\circ} \mathrm{S} / 43^{\circ} \mathrm{O}$. Parte do leste e norte do Nordeste há uma circulação anticiclônica, que dificulta a formação de nebulosidade. No dia 30/10/2015 às 00:00 UTC (Figura 8-b), o continente permanece sob influência de uma a área anticiclônica mencionada na análise do dia anterior, porém uma circulação anticiclônica adentra o continente. Este sistema ou circulação gera subsidência do ar e dificulta a formação de nebulosidade em parte da Região Sul (nordeste de SC e leste, nordeste, centro e norte do PR), principalmente. Um cavado atua entre o AC, leste da Bolívia, Paraguai até oeste do RS, que favorece o levantamento do ar gerando nebulosidade convectiva em algumas áreas a leste dessas áreas. Um Vórtice Ciclônico é visto no leste do Sudeste do Brasil em torno de $20^{\circ} \mathrm{S} / 41^{\circ} \mathrm{O}$.

O campo de umidade relativa e temperatura no nível de $850 \mathrm{hPa}$ da 00UTC do dia 29/10/2015 é ilustrado na Figura 9-a, apresenta uma circulação anticiclônica que atua na Região Sul do Brasil e Atlântico adjacente. Notam-se ventos fortes de norte entre a Bolívia e o leste 

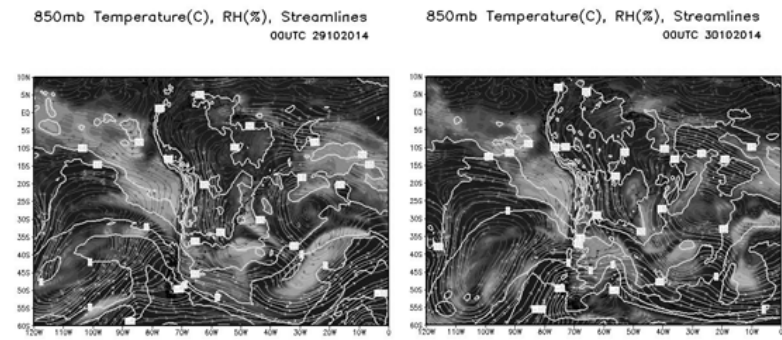

(a)

Figura 9: Campo de umidade relativa, temperatura e linhas de corrente em $850 \mathrm{hPa}$, (a) 29/10/2014 00:00 UTC (b) 30/10/2014 00:00 UTC.

da Argentina e o oeste do RS, evidenciando a presença do Jato de Baixos Níveis (JBN). Esta circulação transporta ar quente e úmido de norte para estas áreas, favorecendo assim a instabilidade. No Pacífico o Anticiclone Subtropical do Pacífico Sul (ASPS) está localizado a oeste de $75^{\circ} \mathrm{O}$ e entre $20^{\circ} \mathrm{S}$ e $30^{\circ} \mathrm{S}$. Um cavado atua entre o sul do Chile e para noroeste no Pacífico até $32^{\circ} \mathrm{S} / 85^{\circ} \mathrm{O}$. Um cavado frontal atua no Atlântico nas proximidades de $40^{\circ} \mathrm{O}$ e entre $37^{\circ} \mathrm{S}$ e $47^{\circ} \mathrm{S}$. A isoterma de zero grau atua a sul de $44^{\circ} \mathrm{S}$ no continente. Na análise da 00 UTC do dia 30/10/2015 (Figura 9 -b), nota-se a atuação da Alta Subtropical do Atlântico Sul (ASAS), que contribui para os ventos fortes de leste seu centro está localizado em torno de $28^{\circ} \mathrm{S} / 28^{\circ} \mathrm{O}$. No oeste desta circulação é possível observar a presença do Jato de Baixos Níveis (JBN), que favorece a produção de temporais em parte do Sul do Brasil, principalmente sobre o RS. Esta circulação transporta ar quente e úmido de norte para estas áreas e favorece a instabilidade.

\subsection{Prognósticos e Análise}

O impacto da assimilação de dados 4DVAR é avaliado sobre as variáveis de refletividade, precipitação e temperatura. O campo de refletividade foi analisado espacialmente e comparado com os dados de radar do DCEA, para a comparação espacial do campo de precipitação foi utilizado dados do TRMM. Os valores pontuais (sobre estações de coleta de dados) de precipitação e temperatura foram avaliados e comparados com os dados observados das estações automáticas do INMET.

Segundo dados da mídia eletrônica, entre os dias 29 e 30 de outubro de 2014, algumas cidades no Oeste e Sul do Rio Grande do Sul apresentaram níveis de precipitação para um intervalo de 12 horas em torno de 97 milímetros (FONTE: CLIMATEMPO). Analisando espacialmente os resultados para este período e comparando com os dados inferidos pelo TRMM Figura 10-a, foi possível observar que tanto a simulação WRF, represen-
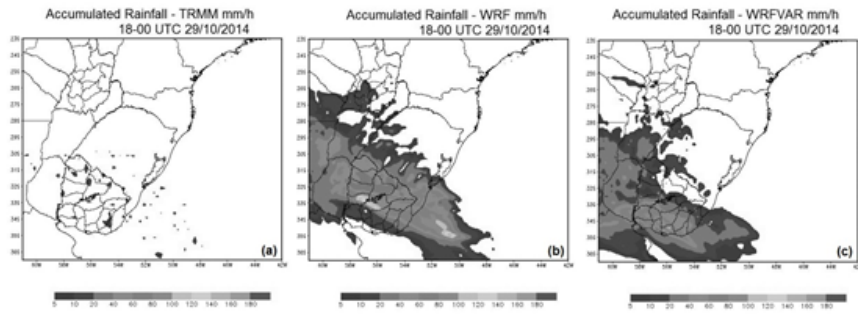

Figura 10: Campos de precipitação acumulada em 12 horas do dia 29/10/2014 às 18:00 UTC ao dia 30/10/2014 às 06:00 UTC para (a) TRMM (b) WRF (c) WRFVAR.
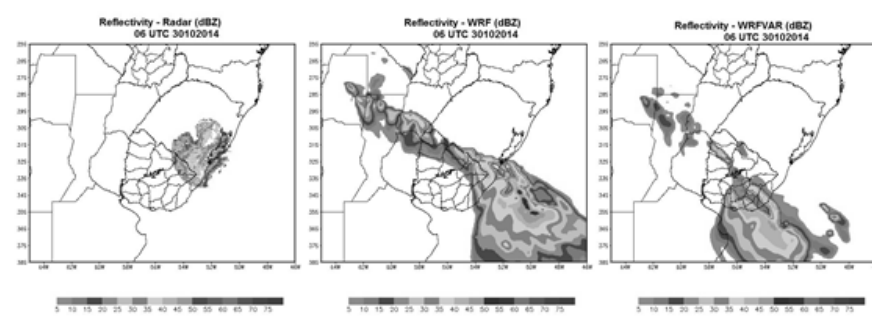

Figura 11: Campos de Refletividade do dia 30/10/2014 às 06:00 UTC (a) Radar (b) WRF (c) WRFVAR.

tada pela Figura 10-b, quanto à simulação WRFVAR (Figura $10-\mathrm{c}$ ) superestimaram os valores de precipitação. Ressalta-se que o TRMM é um satélite desenvolvido para a região tropical e apresenta na maioria das vezes uma frequência temporal incompatível com a ocorrência das precipitações, justificando assim os poucos núcleos de precipitação na figura do TRMM para o período de interesse. Ainda na Figura 10 -b nota-se que o modelo WRF sem a inclusão de assimilação de dados detectou com clareza, em vários pontos do Estado do Rio Grande do Sul, precipitações em torno de $80 \mathrm{~mm}$.

O campo de refletividade observado para o dia 30 de outubro de 2014 às 06:00 UC é apresentado na Figura 11-a. Observa-se que em grande parte do domínio apresenta valores entre $25 \mathrm{dBZ}$ caracterizando intensidade fraca e $45 \mathrm{dBZ}$, que representa intensidade forte. Esses valores em escala de intensidade de precipitação representam intensidade entre 1.5 a $3 \mathrm{~mm}$ e 25 a $50 \mathrm{~mm}$, respectivamente. Na Figura 11-b, tem-se o campo de refletividade sem assimilação de dados. A oeste e sul do Estado do Rio Grande do Sul é possível observar vários pontos valores entre $25 \mathrm{dBZ}$ e $45 \mathrm{dBZ}$, pontos esses que coincidem com aqueles apresentado na Figura 11-a. O campo de refletividade com assimilação de dados da Figura 11-c, não foi capaz de prognosticar os valores de refletividade. Analisando as simulações para o período de 48 horas, constatou-se que o WRFVAR apresentou uma defasagem de aproximadamente 3 horas na representação do campo de refletividade.

A previsão de 48 horas, compreendida entre às 12:00 


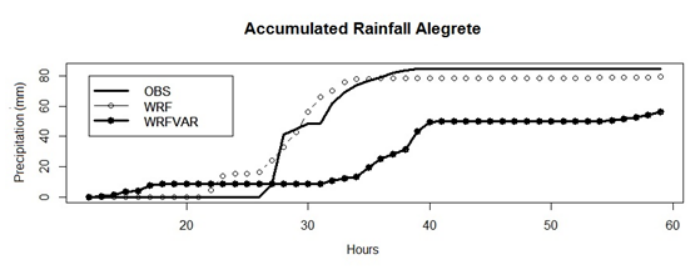

Figura 12: Evolução temporal da precipitação para a cidade de Alegrete.

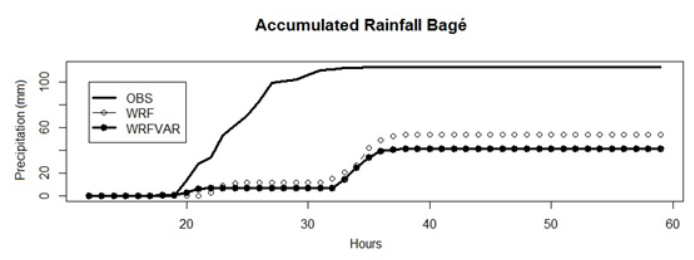

Figura 13: Evolução temporal da precipitação para a cidade de Bagé.

UTC do dia 29 de outubro de 2014 às 12:00 UTC do dia $31 / 10 / 2014$, foi avaliada para as cidades que indicaram um volume maior de precipitação no período. Segundo dados das estações automáticas do INMET a cidade de Alegrete, Bagé, Dom Pedrito e Rio Grande registraram valores próximos ou superiores a $80 \mathrm{~mm}$. Na Figura 12 , onde em 48 horas a precipitação acumulada em Alegrete atingiu um volume de $84,5 \mathrm{~mm}$, o modelo WRF sem assimilação de dados apresentou um desempenho coerente com o observado. A simulação WRFVAR nas primeiras 12 horas de simulação superestima os volumes de precipitação em relação ao observado e ao WRF, já nas 36 horas posteriores ele subestima esses valores.

Na cidade de Bagé, conforme indicado na Figura 13, o volume de precipitação em 36 horas foi de $113 \mathrm{~mm}$ valor este, que segundo as normais climatológicas do INMET, superou a média mensal do ano de 2013 em torno de $57 \mathrm{~mm}$. As simulações WRF e WRFVAR subestimaram os valores para essa região em relação à precipitação observada. Entretanto as evoluções temporais do WRF e do WRFVAR apresentaram com valores bem próximos e, em alguns momentos foram igualadas. A simulação

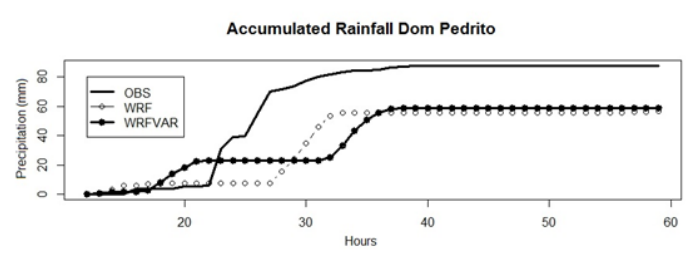

Figura 14: Evolução temporal da precipitação para a cidade de Dom Pedrito.

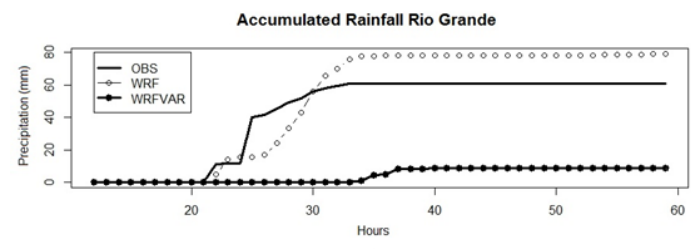

Figura 15: Evolução temporal da precipitação para a cidade de Rio Grande.

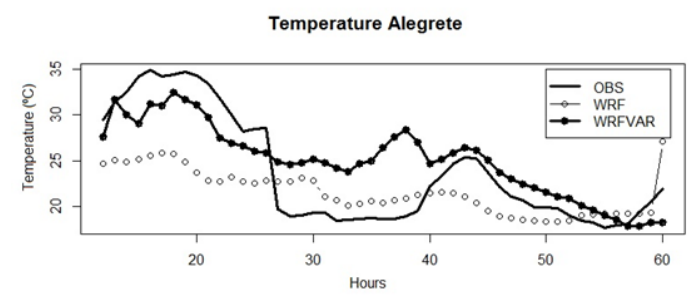

Figura 16: Evolução temporal da temperatura para a cidade de Alegrete.

da evolução temporal para a cidade de Dom Pedrito apresentada na Figura 14, mostra que para o intervalo compreendido entre as 18:00 UTC e 22:00 UTC a simulação sem assimilação de dados (WRF) teve valores bem próximos em alguns momentos iguais ao observado, enquanto o WRFVAR superestimou esses valores. Posteriormente ambas as simulações subestimam os valores em relação ao observado. Na cidade de Rio Grande (Figura 15) foi onde o modelo com assimilação teve o pior desempenho, pois apresentou grande discrepância em relação ao dado observado. A evolução temporal da temperatura para as cidades de Alegrete, Bagé, Dom Pedrito e Rio Grande são apresentadas pelas Figuras 16, 17, 18 e 19, respectivamente. Para a cidade de Alegrete ( 16), é possível observar que o WRFVAR acompanha em quase todo o percurso a curva da temperatura observada, exceto no momento em que há maior intensidade de precipitação que o mesmo mantém as temperaturas um pouco mais elevadas. Entretanto seu desempenho foi considerado superior em relação à simulação sem

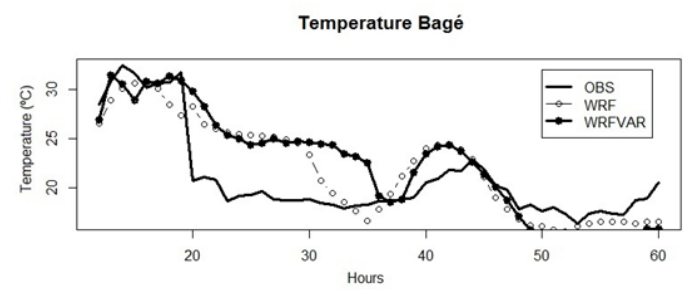

Figura 17: Evolução temporal da temperatura para a cidade de Bagé. 


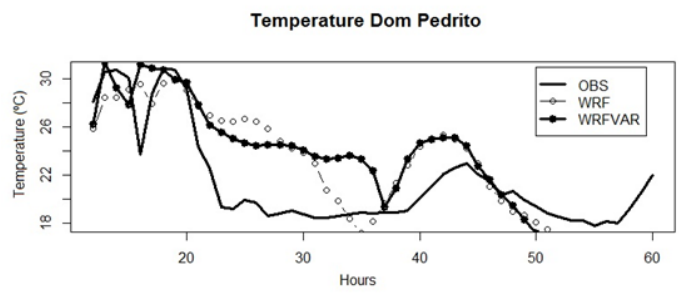

Figura 18: Evolução temporal da temperatura para a cidade de Dom Pedrito.

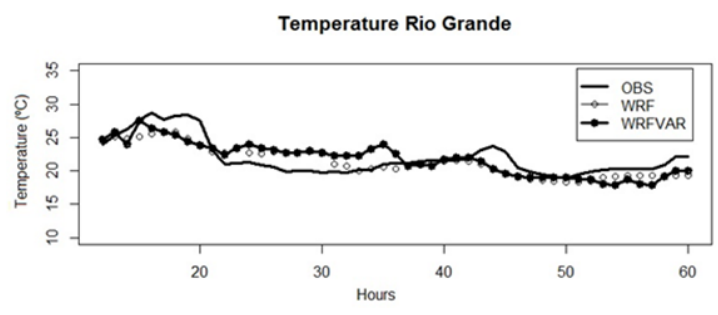

Figura 19: Evolução temporal da temperatura para a cidade de Rio Grande.

assimilação de dados. A Figura 17 mostra a evolução temporal da temperatura para a cidade de Bagé. As simulações, com ou sem assimilação, apresentam valor de máximo bem próximo, ou até mesmo igual ao observado nas primeiras 10 horas de processamento. Ao se estender o horizonte de integração surge uma diferença considerável entre as simulações e os valores observados, mas ainda assim os prognósticos acompanharam as inversões de temperatura no decorrer do tempo. Para a cidade de Dom Pedrito, as simulações apresentaram desempenho semelhante ao descrito para a cidade de Bagé, porém em alguns momentos é possível observar que em determinados instantes (intervalo 30-40 horas), as simulações apresentam um pico de temperatura mínima inexistente nos dados observados, conforme pode ser observado na Figura 18. As simulações para a cidade de Rio Grande (Figura 19) foi a que obteve maior coerência numérica com os dados observados, ou seja, as diferenças de temperaturas foram mínimas e tanto a simulação do WRF quanto a do WRFVAR acompanharam o desenvolvimento da curva observada.

\section{Conclusões}

Para avaliar o impacto da assimilação de dados meteorológicos de superfície no modelo de mesoescala WRF, em um evento de precipitação intensa no Rio Grande do Sul, foi utilizado o sistema assimilação de dados WRFDA/4DVAR. Inicialmente o modelo WRF foi rodado com dados do modelo global GFS, para gerar o campo de background. Posteriormente realizou-se a assimilação de dados utilizando o campo de background com vários conjuntos de dados (dado do Sistema Global de Telecomunicações (GTS) e estações automáticas). Os resultados foram inseridos como condição inicial no modelo WRF, para gerar prognósticos do tempo. As principais variáveis analisadas precipitação e refletividade.

Analisando espacialmente os resultados para o período de 12 horas, compreendido entre os dias 29 e 30 de outubro de 2014, onde algumas regiões do Estado apresentaram acumulados em torno de $97 \mathrm{~mm}$, e comparando com os dados inferidos pelo TRMM foi possível observar que tanto a simulação WRF, quanto a simulação WRFVAR superestimaram os valores de precipitação. Entretanto, o modelo sem assimilação de dados prognosticou com destreza vários núcleos de precipitação com valores próximos a $80 \mathrm{~mm}$, e ambas as simulações foram capazes de detectar as áreas com ocorrência de precipitação.

Na comparação dos campos de refletividade observado para o dia 30 de outubro de 2014 às 06:00 UTC com os campos simulados WRF e WRFVAR, constatou-se que o campo sem assimilação de dados WRF, foi capaz de detectar com melhor precisão núcleos de refletividade sobre o Estado do Rio Grande do Sul, contrastando como o fato de que o campo com assimilação de dados não apresentou uma boa precisão para este campo.

A análise espacial do campo de precipitação acumulada, compreendida entre às 12:00 UTC do dia 29 de outubro de 2014 às 12:00 UTC do dia 31/10/2014, foi comparada com o produto 3B42 do TRMM, onde a simulação do modelo WRF sem assimilação de dados apresentou um desempenho coerente com o observado. A simulação WRFVAR nas primeiras 12 horas de simulação superestima os volumes de precipitação em relação ao observado e ao WRF e nas 36 horas posteriores subestima esses valores.

Para a variável de temperatura, ambas as simulações apresentaram um desempenho significativo, porém constata-se melhora na previsão com a assimilação de dados.

\section{Referências}

CPTEC (2015). Centro de pesquisas e estudos climáticos. URL http://www. satelite.cptec. inpe.br/home.

De Gonçalves, L. G. G., Shuttleworth, W. J., Nijssen, B., Burke, E. J., Marengo, J. A., Chou, S. C., Houser, P., Toll, D. L. (2006). Evaluation of model-derived and remotely sensed precipitation products for continental south america. Journal of Geophysical Research, 111.

Kalnay, E. (2003). Atmospheric Modeling, Data Assimilation and Predictability. Cambridge University Press. 
Lorenc, A. C. (1986). Analysis methods for numerical weather prediction. Quarterly Journal of Royal Meteorology Society, 112(474), 1177-1194.

Macedo, L. R., Alves, R. C. M., Basso, J. L. M., Yamasaki, Y. (2014). Análise do desempenho do modelo wrf com alta resolução espacial na previsão de precipitação acumulada. Ciência e Natura, Edição Especial, 497-502.

Mitra, A. K., Bohra, A. K., Rajeevan, M. N., Krishnamurti, T. N. (2009). Daily indian precipitation analysis formed from a merge of rain-gauge data with the trmm tmpa satellite-derived rainfall estimates. Journal of the Meteorological Society of Japan, 87A, 265-279.

REDEMET (2015). URL http://www.redemet.aer.mil. br.

Rozante, J. R., Cavalcanti, I. F. A. (2008). Regional eta model experiments: Salljex and mcs development. Journal of Geophysical Research, 113.

Rozante, J. R., Moreira, D. S., De Gonçalves, L. G. G., Vila, D. A. (2010). Combining trmm and surface observation precipitation: Technique and validation over south america. Weather and Forecasting, 25, 885-894.

Skamarock, J. B., W.C.and Klemp, Dudhia, J., Gill, D. O., Barker, D. M., Duda, H. X., M. G., Wang, W., Powers, J. G. (2010). A Description of the Advanced Research WRF Version 3. National Center for Atmospheric Research, Boulder, Colorado, United States of America, URL http://www2.mmm.ucar.edu/wrf/ user/docs/arw_v3.pdf. 